\title{
Current practices around HIV disclosure to children on highly active antiretroviral therapy
}

\author{
G D Naidoo, ${ }^{1} \mathrm{MB}$ ChB, MMed (Paediatrics and Child Health), DCH (SA), FCPaed (SA); \\ N H McKerrow, ${ }^{1,2}$ MB ChB, BA, DCH (SA), FCPaed (SA), MMed Paed, PG Dip Int Research Ethics \\ 'Department of Paediatrics, Nelson Mandela School of Medicine, College of Health Sciences, University of KwaZulu-Natal, Durban, \\ South Africa \\ ${ }^{2}$ KwaZulu-Natal Provincial Department of Health, Durban, South Africa
}

Corresponding author: G Naidoo (gitanya.naidoo@gmail.com)

\begin{abstract}
Background. The introduction of antiretroviral therapy (ART) for children has resulted in survival into adolescence. This is associated with the challenge of disclosing HIV status to infected children.

Objectives. To establish whether HIV disclosure had occurred, the process of disclosure or reasons for non-disclosure, and the effect of disclosure on the child's understanding of their disease and adherence.

Methods. Interviews were conducted with the caregivers of $100 \mathrm{HIV}$-positive children, aged 8 - 14 years, who were on ART for $>1$ year to determine if disclosure had occurred. Where disclosure had occurred, these children were interviewed.

Results. Disclosure had occurred in only 27 patients. The age and gender of the caregiver and their relationship to the child did not influence the likelihood of disclosure. The educational level of the caregiver and the number of admissions of the child were both associated with disclosure. Disclosure did not improve adherence, as reflected by an increased CD4 count or reduced viral load.

Conclusion. HIV disclosure to children on ART remains less than optimal despite the presence of both national and international guidelines. Caregivers cited fear on the part of the child and fear of being blamed for their illness as the main reasons that they do not disclose. Of the children who knew their status, $76.9 \%$ had already suspected that they had HIV.
\end{abstract}

S Afr J Child Health 2015;9(3)85-88. DOI:10.7196/SAJCH.7957

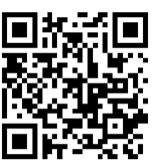

South Africa (SA) remains the epicentre of the global HIV epidemic. The 2009 antenatal HIV seroprevalence survey ${ }^{[1]}$ estimated an HIV prevalence of $29.4 \%$ in pregnant women, with an estimated 5.2 5.63 million HIV-infected adults and children in the country. The Human Sciences Research Council's 2012 household survey ${ }^{[2]}$ estimated an HIV prevalence of $2.4 \%$ in children between the ages of 2 and 14 years and $1.7 \%$ in children $<5$ years of age. This equates to $369000 \mathrm{HIV}$-infected children, of whom 166000 were on antiretrovirals (ARVs) (45.1\%).

Antiretroviral therapy (ART) is critical in the effective care and management of the HIV-infected individual. With the initiation and the increasing roll out of ART, HIV-infected children experience a less symptomatic early course, live longer and have a better quality of life. ${ }^{[3]}$

The extensive use of ART has changed paediatric HIV infection into a chronic disease of childhood. The social difference between this and other chronic childhood diseases lies in the stigma associated with HIV infection. This frequently results in an emotional response from infected individuals upon disclosure of such a diagnosis. ${ }^{[4]}$ As with other diseases, knowledge about the condition and treatment thereof is essential for understanding and accepting the diagnosis. One of the greatest psychosocial challenges that parents and caregivers of HIV-infected children face is disclosing HIV status to their children and enlisting the child's cooperation in treatment programmes. HIV disclosure entails communication about a potentially life-threatening, stigmatised and transmissible illness, and many caregivers fear that such communication may create distress for the child. ${ }^{[5]}$

Research on disclosure to children with cancer showed that prior to the 1970s, children were given little information regarding their condition and prognosis, as it was thought that they had limited understanding about the illness and required protection from the emotional burdens faced by their parents. Recent advances support a more open and communicative approach to children with cancer in light of improved survival rates, increasing advocacy for children's rights and children's participation in the management of their disease. ${ }^{[6]}$

In 1999, the American Academy of Pediatrics ${ }^{[7]}$ released guidelines suggesting that adolescents should know their HIV status and that HIV disclosure should be considered for school-aged children. Local guidelines suggest that disclosure should be an ongoing, progressive process supported by parents and caregivers. ${ }^{[8]}$ These guidelines suggest that disclosure should occur at an age-appropriate level, recognising the child's cognitive and developmental level. It is suggested that partial disclosure occurs between the ages of 8 and 11 years, and full disclosure, assuming better understanding of the HIV disease and treatment options, between 11 and 14 years. Failure to achieve full disclosure by the early teens is associated with poor adherence, emotional difficulties, breakdown of trust and unwitting HIV transmission. ${ }^{[6]}$

\section{Methods}

The study was conducted at the Paediatric ART Clinic, Edendale Hospital, Pietermaritzburg. Participants for the study were recruited over a 2-week period in December 2013 during routine follow-up visits to the clinic.

The caregivers of HIV-infected children aged 8 - 14 years who had completed at least 12 months of ARV treatment were enrolled in the study. Children to whom their HIV status had been disclosed were also enrolled in the study.

Interviews were conducted in the interviewee's home language, using standardised questionnaires specific to the caregiver or the 
child. Three different questionnaires were used. A questionnaire was designed for caregivers who had disclosed to assess when and how disclosure was conducted, and the response of the child to disclosure. A separate questionnaire was used to assess the reasons for non-disclosure by caregivers. Lastly, there was a questionnaire aimed at the children who were aware of their HIV status, to provide information regarding the child's response to disclosure.

Clinical records of all children, irrespective of disclosure, were reviewed with respect to their clinical, immunological and virological status. In children where disclosure was noted, a comparison of these parameters was done pre- and postdisclosure.

Ethical approval for the study was obtained from the Biomedical Research Ethics Committee of the University of KwaZulu-Natal.

Statistical analysis was undertaken with the assistance of the Discipline of Public Health Medicine, University of KwaZulu-Natal, using $\chi^{2}$, Student's $t$-test or the Fisher's exact test. A $p$-value of $<0.05$ was considered statistically significant.

\section{Results}

The caregivers of the first 100 children who met the criteria and consented to participate were enrolled in the study. Of these children, disclosure had occurred in 27 patients, while 73 were unaware of their HIV status.

Characteristics of the caregiver are represented in Table 1. While most caregivers were female, there was a slightly higher number of males in the non-disclosure group; however, this was not statistically significant. The age range was broader in the non-disclosure group while the mean was less; however, this was not significant. The majority of caregivers knew their HIV status and the proportion of HIV-positive caregivers in the groups was similar. The data indicated that caregivers with either no formal education or only primary education were more likely to disclose than those with secondary education.

The characteristics of the children who participated in the study are portrayed in Table 2 . The age range was similar in both groups, and reflected the narrow age range of the selection criteria. The majority of children (77\%) had been on ART for $>5$ years. A slightly higher percentage of children who were on treatment for $<5$ years were disclosed to than those on treatment for $>5$ years, i.e. $30 \%$ and $26 \%$, respectively. Children who had more than five admissions to hospital were $33 \%$ (odds ratio 1.33) ( $p=0.03$ ) more likely to have their status disclosed to them than children with one or two admissions.

Table 3 provides information relating to the process of disclosure to children. Although the mean age of disclosure was 11.6 years, disclosure had occurred in $58 \%$ of children by the age of 10 years. In most instances (84.6\%), the parent disclosed. In the majority of cases $(72.0 \%)$, this was in response to the advice of a healthcare worker. Caregivers conducted just over half $(55.6 \%)$ of these disclosures alone, while the remainder were assisted by the healthcare worker. At the time of disclosure, an equal number of children already knew their HIV status to those who were unaware of their diagnosis. On hearing their HIV status, $7.4 \%$ of the children were afraid.

Every caregiver who had disclosed the HIV status to their child believed that their child had a right to know this status and was happy that they were aware of it. Caregivers who had not disclosed were questioned regarding their rationale for not disclosing (Table 4). The majority $(n=46)$ were afraid of the child's response to the disclosure. Other concerns identified included fear of being blamed by the child for infecting them $(n=8)$, the child being too young to understand $(n=7)$, concern of poor compliance following disclosure $(n=5)$, fear of stigma $(n=4)$, uncertainty of how to go about disclosing $(n=1)$ and the mental competence of the child $(n=1)$.

There was a statistically significant difference in the rationale provided by caregivers according to their relationship to the child. Grandparents did not disclose because they were afraid of the
Table 1. Characteristics of caregivers

\begin{tabular}{llll}
\hline & Disclosed & Non-disclosed & $p$-value \\
\hline Age (years) & & & 0.377 \\
$\quad$ Range & $18-64$ & $19-72$ & \\
Mean (SD) & $43.54(2.43)$ & $40.63(1.81)$ & \\
Gender (female), $n$ (\%) & $23(30.3)$ & $53(69.7)$ & 0.225 \\
Education, $n$ (\%) & & & 0.041 \\
$\quad$ No formal, primary & $13(48.1)$ & $25(34.2)$ & \\
$\quad$ Secondary, tertiary & $14(51.8)$ & $47(64.3)$ & \\
HIV status, $n$ (\%) & & & 0.630 \\
$\quad$ Positive & $11(26.8)$ & $30(73.2)$ & \\
$\quad$ Negative & $14(26.4)$ & $39(73.6)$ & \\
$\begin{array}{l}\text { Unknown/unwilling to } \\
\text { disclose }\end{array}$ & $2(50.0)$ & $2(50.0)$ & \\
& & &
\end{tabular}

Table 2. Profile of children

\begin{tabular}{|c|c|c|c|}
\hline & Disclosed & Non-disclosed & $p$-value \\
\hline Mean age (years) & 11.6 & 10.7 & 0.115 \\
\hline $\begin{array}{l}\text { Duration of treatment } \\
\text { (years), } n(\%)\end{array}$ & & & 0.672 \\
\hline$<5$ & $7(30.4)$ & $16(69.6)$ & \\
\hline$\geq 5$ & $20(25.9)$ & $57(74.1)$ & \\
\hline $\begin{array}{l}\text { Number of hospital } \\
\text { admissions, } n(\%)\end{array}$ & & & 0.030 \\
\hline $1-2$ & $13(24.5)$ & $24(72.7)$ & \\
\hline $3-5$ & $9(27.3)$ & $40(75.5)$ & \\
\hline$>5$ & $4(33.3)$ & $8(66.7)$ & \\
\hline
\end{tabular}

Table 3. Process of disclosure

Age at disclosure (years), mean (SD)

Person responsible for disclosure, \% ( $n$ )

Parent

$84.6(22)$

Grandparent

$11.5(3)$

Nurse

Process of disclosure, \% ( $n$ )

Alone

With assistance

Reason for disclosure

Requested to disclose by healthcare worker

Child started asking questions about their illness

Child's response, \% ( $n$ )

Already knew

$40.7(11)$

Did not understand

$40.7(11)$

Afraid

$7.4(2)$

Angry 


\begin{tabular}{ll} 
Table 4. Profile of non-disclosure & \\
\hline Reason for not disclosing, $\%(n)$ & $63.0(46)$ \\
Fear of child's response & $11.0(8)$ \\
Fear of blame & $5.5(4)$ \\
Fear of stigma & $20.5(15)$ \\
Other & $10.7(0.33)$ \\
Planned age of disclosure (years), mean (SD) & \\
Planned person responsible for disclosure, $\%(n)$ & $19.2(14)$ \\
Caregiver & $65.8(48)$ \\
Members of the family & $15.0(11)$ \\
Healthcare workers & \\
Process of disclosure, $\%(n)$ & $21.9(16)$ \\
Alone & $78.1(57)$ \\
With assistance & \\
Anticipated response, $\%(n)$ & $23.3(17)$ \\
Already suspected & $68.5(50)$ \\
Scared & $4.1(3)$ \\
Angry & $4.1(3)$ \\
Suicidal &
\end{tabular}

emotional response of the children in $75 \%$ of cases, while only $45 \%$ of parents were afraid of the child's emotional response. A higher percentage of parents (29\%) were afraid that they would be blamed by their children for their illness compared with $4 \%$ of grandparents.

The average age at which non-disclosing caregivers planned to inform their charge of their HIV status was 10.7 years. Most believed that responsibility for this disclosure lay with the child's parents (84.6\%); a smaller proportion allocated this responsibility to the grandparents (11.5\%) and very few to the healthcare worker $(3.8 \%)$.

Two-thirds of caregivers $(65.8 \%)$ indicated that they would seek assistance from their family when disclosing, 15\% would seek assistance from a healthcare worker and $19.2 \%$ would disclose alone without any assistance.

A quarter of caregivers (23.3\%) believed that their children already suspected that they had HIV, two-thirds (68.5\%) anticipated that their children would be scared at hearing the news, and a minority (4.1\% each) anticipated that their children would either be angry or become suicidal upon hearing the news.

The effect of HIV disclosure on the clinical wellbeing of the child was assessed by reviewing any effect on CD4 levels and HIV viral load (VL). The trend in both these parameters was similar in children in both the disclosed and undisclosed groups. In both groups the CD4 levels increased and the VL declined.

\section{Discussion}

Twenty-seven per cent of children in this study had been formally disclosed to regarding their HIV status. This lies within the range of disclosure reported in other developing countries, e.g. $17.4 \%$ in Ethiopia and 31\% in Zambia, ${ }^{[9]}$ but is three times higher than the $9 \%$ reported in Cape Town. ${ }^{[10]}$ The three-fold variation in disclosure rates within SA may reflect the different periods during which the studies were conducted or a difference in HIV prevalence between these two communities at the times of each study, namely $6.0 \%$ in Cape Town ${ }^{[2]}$ compared with $16.9 \%$ in KwaZulu-Natal.

The age and gender of the caregiver as well as their relationship to the child did not influence whether they were more or less likely to disclose. Only two factors were found to influence disclosure, namely the educational level of the caregiver and the number of admissions of the child to hospital.

We found a higher rate of disclosure among caregivers with a lower level of education. This was in line with reports from Ethiopia, Thailand and Cape Town. A study done in Ethiopia, where education was used as a proxy indicator of higher economic status, suggested that the lower rate of disclosure found in more affluent families resulted from fear that children would inadvertently disclose their HIV status and thereby bring shame on the family. ${ }^{[11]}$

In our experience, where disclosure had occurred this was prompted by a healthcare worker in $72 \%$ of all cases, regardless of the caregivers' educational level. Prompting may have influenced caregivers with a lower level of education to disclose without necessarily considering the broader social consequences of such disclosure, which a caregiver with a higher level of education may have considered as demonstrated in other studies. ${ }^{[1]}$

Children with more than five admissions were found to have a higher rate of disclosure, and in more than half of these (60\%) this was precipitated by questions from the child. This finding has not previously been reported and suggests that children may seek an explanation for poor health.

The mean age at which disclosure occurred in this study was 11.6 years, which is higher than that found in the Cape Town study (9.4 years) but comparable with reports from developed countries, where the mean age ranged from 5 to 14 years. ${ }^{[10]}$

Three-quarters of caregivers had not disclosed HIV status to their child even though the majority (93.2\%) believed that the child has the right to know their HIV status. The reported mean age at which they intended to disclose the HIV status to their child was 10.7 years. This was the mean age of children to whom disclosure had not occurred, which suggests that they had probably not considered disclosure until the issue was raised as part of this study.

Reasons reported for not disclosing were similar to those noted in previous studies, ${ }^{[5]}$ namely fear of the emotional effect on the child, fear of blame for infecting the child and fear of stigma and ostracism. However, in this study there was less emphasis on fear of stigma as a reason for non-disclosure.

The majority of caregivers (78\%) indicated that they would require assistance from a healthcare worker in order to disclose. However in this study, a smaller percentage (44.4\%) of caregivers actually required the assistance of a healthcare worker. Of the caregivers who had disclosed, $55.6 \%$ had done so independently without any assistance, although $72 \%$ only did so after prompting from the healthcare worker or in response to queries from their child.

The response of children to disclosure differed markedly from what caregivers anticipated. Caregivers overestimated the adverse effect of disclosure on the emotional state of the child, with an emphasis on fear $(68.5 \%)$, while in reality a minority of children reported such a reaction $(7.4 \%)$. In contrast, caregivers underestimated how many children already suspected that they were HIV-positive (23.3\%) compared with the number who actually suspected that they were infected (40.7\%).

There was no immediate clinical benefit noted in association with disclosure. Trends in the CD4 count and VLs were similar in children who were aware and who were not aware of their HIV status. This may be explained by the fact that the caregivers assumed responsibility for the collection and administration of treatment to their children and so adherence was more likely to be influenced by the knowledge and behaviour of the caregiver rather than the child.

Mahloko and Madiba ${ }^{[9]}$ found a similar lack of clinical improvement in response to HIV disclosure. Despite this, they suggested that disclosure remains an essential element in ensuring better compliance especially in the face of asymptomatic HIV disease, when there is a risk that children may stop taking their treatment. 
Findings of this study are comparable with those of previous reports, which show low levels of disclosure and an absence of immediate clinical benefit following disclosure. In addition, this study found that caregivers underestimate the knowledge and suspicion of their children and place undue emphasis on the emotional effect of disclosure on the child.

\section{Conclusion}

Despite recommendations from the American Academy of Paedia$\operatorname{trics}^{[4]}$ and local guidelines promoting the disclosure of HIV status to children, this remains a difficult and controversial issue with low rates of disclosure. ${ }^{[5]}$ These low rates are underpinned by caregivers' fear of the emotional effect of disclosure on the child and fear of being blamed for the illness. Despite efforts to protect children from the knowledge of their disease, most children suspected that they had the virus due to frequent admissions and the need for daily treatment.

Greater emphasis is required to encourage disclosure to older children. This should be facilitated by healthcare professionals during routine clinic visits. Locally available guidelines outline the process of progressive disclosure to ensure full disclosure by adolescence, with comprehensive understanding of the illness, the need for treatment compliance and the dangers of high-risk behaviour and transmission. HIV disclosure should therefore become an integral aspect in the comprehensive care for every child on ART.

\section{References}

1. Department of Health. National Antenatal Sentinel HIV and Syphilis Seroprevalence Survey, 2009. Pretoria: Department of Health, 2010.

2. Shisana O, Rehle T, Simbayi LC, et al. South African National HIV Prevalence, Incidence and Behaviour Survey, 2012. Cape Town: Human Sciences Research Council Press, 2014.

3. Butler A, Williams P, Howland L, Storm D, Hutton N, Seage G. Impact of disclosure of HIV on health-related quality of life among children and adolescents with HIV Infection. Paediatr 2009;123(3):935-943. [http://dx.doi. org/10.1542/peds.2008-1290]

4. Naeem-Sheik A, Gray G. HIV disclosure in children. S Afr J HIV Med 2005;6(4):46-48.

5. Vaz ME, Eng E, Maman S, Tshikandu T, Behets F. Telling children they have HIV: Lessons learned from findings of a qualitative study in sub-Saharan Africa. AIDS Patient Care STDs 2010;24(4):247-256. [http://dx.doi.org/10.1089/ apc.2009.0217]

6. Wiener L, Mellins CA, Marhefka S, Battles HB. Disclosure of an HIV diagnosis to children: history, current research and future directions. J Dev Behav Pediatr 2007;28(2):155-166. [http://dx.doi.org/10.1097/01.DBP.0000267570.87564.cd]

7. American Academy of Paediatrics Committee on Paediatrics AIDS. Disclosure of illness status to children and adolescence with HIV infection. Paediatrics 1999;103(1):164-166.

8. McKerrow NH, Stephen CR, Purchase SE, et al. Step-by-step Guide for the Management of Children on ART. 4th ed. Pietermaritzburg: UNICEF, 2010.

9. Mahloko JM, Madiba S. Disclosing HIV diagnosis to children in Odi district, South Africa: Reasons for disclosure and non disclosure. Afr J Prim Healthcare Fam Med 2012;4(1):345-352. [http://dx.doi.org/10.4102/phcfm.v4i1.345]

10. Mokwena K, Mahloko J, Madiba S. Prevalence and factors associated with disclosure of HIV diagnosis to infected children receiving antiretroviral treatment in public health care facilities in Gauteng, South Africa. J Clin Res HIV/AIDS Prev 2014;1(1):35-45.

11. Biadgilign S, Derubrew A, Amberbir A, Escudero, HR, Deribe K. Factors associated with HIV/AIDS diagnostic disclosure to HIV infected children receiving HAART: A multicentre Study in Adis Ababa, Ethiopia. PLoS ONE 2011;6(3). 